CONFORMAL GEOMETRY AND DYNAMICS

An Electronic Journal of the American Mathematical Society

Volume 8, Pages 158-166 (November 16, 2004)

S $1088-4173(04) 00115-8$

\title{
EXTENDING RATIONAL MAPS
}

\author{
GAVEN J. MARTIN
}

\begin{abstract}
We investigate when a rational endomorphism of the Riemann sphere $\overline{\mathbb{C}}$ extends to a mapping of the upper half-space $\mathbb{H}^{3}$ which is rational with respect to some measurable conformal structure. Such an extension has the property that it and all its iterates have uniformly bounded distortion. Such maps are called uniformly quasiregular. We show that, in the space of rational mappings of degree $d$, such an extension is possible in the structurally stable component where there is a single (attracting) component of the Fatou set and the Julia set is a Cantor set.

We show that generally outside of this set no such extension is possible. In particular, polynomials can never admit such an extension.
\end{abstract}

\section{INTRODUCTION}

Let $\mathcal{R}_{d}$ denote the space of all degree $d$ rational endomorphisms of the Riemann sphere $\overline{\mathbb{C}}=\mathbb{C} \cup\{\infty\}$. We use [1] and [2] as basic references for the theory of iteration of rational mappings. As usual, we view $\overline{\mathbb{C}}$ as the boundary of the upper half-space $\mathbb{H}^{3}=\left\{\left(x_{1}, x_{2}, x_{3}\right) \in \mathbb{R}^{3}: x_{3}>0\right\}$. Given a rational mapping $R \in \mathcal{R}_{d}$ we say that $\hat{R}: \overline{\mathbb{H}^{3}} \rightarrow \overline{\mathbb{H}^{3}}$ is an extension of $R$ if:

(1) $\hat{R} \mid \overline{\mathbb{C}}=R$;

(2) $\operatorname{degree}(\hat{R})=\operatorname{degree}(R)=d$.

Condition (2) is largely redundant. Whenever an extension has any reasonable topological properties, for instance if it is continuous and open, it will follow.

Rickman has shown that every rational mapping of $\overline{\mathbb{C}}$ admits a quasiregular extension to $\mathbb{H}^{3}[15]$; however, in this paper we investigate which rational mappings admit an extension to $\mathbb{H}^{3}$ which is conformal (or rational) in some measurable conformal structure. Such an extension will have the remarkable property that it and all its iterates are quasiregular with a uniform bound on the distortion (see [14] and [7] for basics concerning quasiregular mappings). Such mappings are known as uniformly quasiregular mappings, abbreviated to uqr-mappings. The existence of such (non-injective) mappings was established in [6]. More recent examples of Lattès type were given by V. Mayer [12]. Largely because of Rickman's version of Montel's Theorem [14, the dynamics associated with iterating a uqr-mapping of $\overline{\mathbb{H}^{3}}$ are quite analogous to the planar situation (see [5], 9], and [12]).

Received by the editors April 15, 2002 and, in revised form, February 1, 2003.

2000 Mathematics Subject Classification. Primary 30C60, 30C65, 30F40, 30D50.

Key words and phrases. Rational mapping, quasiconformal, quasiregular, extension.

Research supported in part by grants from the Australian Research Council, the Marsden Fund and Royal Society (NZ) and Institute Mittag-Leffler (Sweden).

(C)2004 American Mathematical Society Reverts to public domain 28 years from publication 
We shall usually consider an extension of a map of $\mathbb{H}^{3}$ to be defined on $\mathbb{S}^{3}$ via reflection across $\overline{\mathbb{C}}=\partial \mathbb{H}^{3}$ and the conformal identification (stereographic projection) between $\overline{\mathbb{R}}^{3}=\mathbb{R}^{3} \cup\{\infty\}$ and $\mathbb{S}^{3}$. In the literature, such mappings are sometimes called quasimeromorphic [10].

The extension of a rational mapping of $\overline{\mathbb{C}}$ to a $u q r$-mapping of $\overline{\mathbb{R}}^{3}$, such as that given here, opens up the possibility of using 3-dimensional topology as a tool in studying the dynamics of such rational mappings somewhat in analogy with the theory of Kleinian groups. Although in view of the severe nonexistence results we prove, this extension seems to be of limited utility.

For higher $(n \geq 3)$ dimensional quasiregular mappings, the Liouville Theorem, 14] Theorem 2.5, asserts that no extension can be conformal in the usual sense. Moreover, at points of continuity of a measurable conformal structure, a rational mapping is locally injective [7]. Thus we must utilise structures which are at best measurable. The connections between $u q r$-mappings and mappings rational with respect to a measurable conformal structure is discussed in [6]. We give only the briefest sketch below.

We refer to the subset of all $R \in \mathcal{R}_{d}$ with the property that there is a single component of the Fatou set as the unbounded component. Following Mañè-SadSullivan, we say a rational mapping $R \in \mathcal{R}_{d}$ is structurally stable if there are no relations between critical points [11]. Mañè-Sad-Sullivan proved that the set of structurally stable degree $d$ rational mappings is open and dense. We denote by $\mathcal{S}_{d}^{\infty}$ the structurally stable degree $d$ rational mappings whose Fatou set consists of a single component (and whose Julia set is a Cantor set). The set $\mathcal{S}_{d}^{\infty}$ is connected and the theory of holomorphic motions can be used to show that any two mappings in $\mathcal{S}_{d}^{\infty}$ are conjugate by a quasiconformal homeomorphism of $\overline{\mathbb{C}}[11$.

\section{Conformal structures}

We consider $\mathbb{S}^{3}$ as a Riemannian 3-manifold with the usual metric induced by the inclusion $\mathbb{S}^{3} \stackrel{i}{\hookrightarrow} \mathbb{R}^{4}$.

Definition. A mapping $F: \mathbb{S}^{3} \rightarrow \mathbb{S}^{3}$ of Sobolev class $W^{1,3}\left(\mathbb{S}^{3}\right)$ is said to be $K$ quasiregular, $1 \leq K<\infty$ if:

- $J_{F}(x) \geq 0$ almost everywhere,

- $\max _{|\xi|=1}|D F(x) \xi| \leq K \min _{|\xi|=1}|D F(x) \xi|$

for almost every $x \in \mathbb{S}^{3}$, and all $\xi \in T_{x} \mathbb{S}^{3}$.

The smallest number $K$ for which the above inequality holds is called the distortion of $F$. A quasiregular mapping can be redefined on a set of measure zero so as to be continuous, open, and discrete.

Let $\mathbf{G}$ be a measurable conformal structure on $\mathbb{S}^{3}$. By this we mean that at each point $x \in \mathbb{S}^{3}, \mathbf{G}(x)$ is a linear automorphism

$$
\mathbf{G}(x): T_{x} \mathbb{S}^{3} \rightarrow T_{x} \mathbb{S}^{3}
$$

of the inner product space $T_{x} \mathbb{S}^{3}$, such that $\mathbf{G}(x)$ is symmetric, positive definite, of determinant equal to 1 and satisfies a uniform ellipticity condition

$$
K^{-1}|\xi|^{2} \leq\langle\mathbf{G}(x) \xi, \xi\rangle \leq K|\xi|^{2}
$$

with $K \geq 1$ independent of $x$. 
For $\mathbf{G}$ satisfying (2) the solutions of the equation

$$
D^{t} F(x) \mathbf{G}(F(x)) D F(x)=J_{F}(x)^{2 / 3} \mathbf{G}(x)
$$

for mappings of Sobolev class $W^{1,3}\left(\mathbb{S}^{3}\right)$ form a semigroup under composition. Each such solution is a $K^{2}$-quasiregular mapping of $\mathbb{S}^{3}$ where $K$ is determined by the ellipticity bound (2) on $\mathbf{G}$.

We call the semigroup of nonconstant solutions to (3) the G-rational mappings, or simply rational mappings if $\mathbf{G}$ is understood. $\mathbf{G}$ is referred to as an invariant conformal structure for this semigroup.

When $n=2$ and $\mathbf{G}=I$ (the identity matrix) the differential equation (3) reduces to the usual Cauchy-Riemann equations and such solutions necessarily represent rational (analytic) endomorphisms of $\overline{\mathbb{C}}$. Actually, in dimension 2, rather more is true. Any measurable $\mathbf{G}$ can be the matrix dilatation of a quasiconformal homeomorphism (the so-called measurable Riemann mapping theorem).

Let $f: \mathbb{S}^{3} \rightarrow \mathbb{S}^{3}$ be a mapping. We denote the iterates of $f$ by

$$
f^{1}(x)=f(x), \quad f^{n+1}(x)=f\left(f^{n}(x)\right) .
$$

If $f$ is a G-rational mapping, then so is $f^{n}$ for every $n=1,2,3, \ldots$ and the degree of $f^{n}$ is $d^{n}$ where $d$ is the degree of $f$. Conversely, if $f$ is a $u q r$-mapping, then there is an equivariant conformal structure for the semigroup $\left\{f^{n}\right\}$ generated by $f$ [6]. This is not the case for more general semigroups of quasiregular mappings, even if there is a uniform distortion bound [3].

The branch set of a quasiregular mapping $f$ is the set of points $B_{f}$ at which $f$ is not locally injective. The branch set is analogous to the set of critical points; however, G-rational mappings will not be smooth on the branch set in dimensions greater than 2. The branch set of a $u q r$-mapping can be more or less arbitrary in the sense that one cannot distinguish between a quasiregular mapping and a $u q r$-mapping by the structure of the branch set alone [8].

\section{Extensions}

In this section we show how to extend rational mapping in $\mathcal{S}_{d}^{\infty}$. In fact we achieve a slightly more general result. The construction is not too distant from that given in [8] and [7] and so we do not give too many details. We start with a definition.

Let $R$ be a rational mapping of $\overline{\mathbb{C}}$. An inverse disk system for $R$ is a collection of closed topological disks $D_{0}, D_{1}, \ldots, D_{m}$ in the plane with the following properties.

(1) $D_{i} \cap D_{j}=\emptyset$ if $i \neq j$.

(2) $R$ is injective on each $D_{i}$.

(3) There is $N \geq 1$ such that

$$
R^{N}\left(\overline{\mathbb{C}} \backslash \bigcup_{k=1}^{m} D_{k}\right) \subset D_{0} .
$$

(4) $R\left(D_{0}\right) \subset \operatorname{int}\left(D_{0}\right)$.

Obviously the existence of an inverse disk system is a topological property, in the sense that it is preserved under topological conjugacy. We call this system an inverse system of disks because it is what one should obtain by looking at the inverse iterates of a small disk about the attracting fixed point of a rational map whose Julia set is a Cantor set. The disk $D_{0}$ contains the attracting fixed point; the Julia 
set lies in the disks $D_{1}, \ldots, D_{n}$ and is the inverse limit of the disks $R^{-n}\left(D_{i}\right)$. In particular, the Fatou set of a rational mapping with an inverse system is connected and there is a unique attracting fixed point.

The construction given in the proof of Theorem 9.8.1 of Beardon [1] shows (with minor modification) that if $R$ is a rational mapping all of whose critical points lie in the immediate basin of an attracting fixed point, then $R$ admits an inverse disk system. The converse is clear. Note that polynomial mappings can never have an inverse disk system as $\infty$ is always a super-attracting fixed point and therefore Condition (2) is violated on $D_{0}$.

Theorem 3.1. Let $R$ be a rational mapping of $\overline{\mathbb{C}}$ which admits an inverse disk system. Then $R$ has an extension $\hat{R}: \mathbb{H}^{3} \rightarrow \mathbb{H}^{3}$ which is uniformly quasiregular and hence rational in some measurable conformal structure.

Proof. We break the proof into a number of simple steps.

Step 1. We may assume by approximation that all the disks in the inverse system are quasidisks. The images of these disks is also a disjoint collection of quasidisks. Next, appealing to the planar annulus theorem for quasiconformal mappings, there is a quasiconformal mapping $f_{1}: \overline{\mathbb{C}} \rightarrow \overline{\mathbb{C}}$ such that both $D_{i}$ and $R\left(D_{i}\right)=D_{i}^{\prime}=f_{1}\left(D_{i}\right)$ are round circles. Set $R_{1}=f_{1} \circ R \circ f_{1}^{-1}$. Then $R_{1}$ is a mapping (not necessarily rational) for which we have an inverse system of round disks (which we continue to denote by $D_{i}$ ) and for which the iterates $R_{1}^{n}=f_{1} \circ R^{n} \circ f_{1}^{-1}$ are uniformly quasiregular.

Step 2. We use the (planar) Schönfliess theorem in a neighbourhood $U_{i}$ of each disk (chosen so as to be disjoint from all the other disks) to change the mapping $R_{1}$ to a mapping $R_{2}$ with the property that $R_{1}=R_{2}$ outside these small neighbourhoods of disks $\bigcup U_{i}$ and such that $R_{2}: D_{i} \rightarrow D_{i}^{\prime}$ is a linear fractional transformation. We may easily retain Condition (3) so that Condition (4) implies that the mapping $R_{2}$ is uniformly quasiregular. (To see this, note that the forward orbit of any point spends only $N$ iterations outside the disks $D_{i}$ where $R_{2}$ is conformal, and thus picks up at most a finite amount of distortion.) In fact, note that a planar uqr-mapping is conjugate to a rational mapping [6]. (This is because of the existence theorem for quasiconformal mappings which implies that any invariant conformal structure is conformally flat.) This rational mapping will in turn be quasiconformally conjugate to $R$. So, at present, $R_{2}$ is simply a well chosen quasiconformal conjugate of $R$,

$$
R_{2}=f_{2} \circ R \circ f_{2}^{-1} .
$$

Step 3. Let $B_{i}$ (centered in $\mathbb{C}$ ) be the 3 -ball in $\overline{\mathbb{R}}^{3}$ whose intersection with $\overline{\mathbb{C}}$ is the disk $D_{i}$, and similarly $B_{i}^{\prime}$. Let $\phi_{i}: B_{i} \rightarrow B_{i}^{\prime}$ be the Möbius transformation whose restriction to $D_{i}$ is $R_{2}$. We claim there is an extension $\hat{R}_{2}: \overline{\mathbb{R}}^{3} \rightarrow \overline{\mathbb{R}}^{3}$ of $R_{2}$ with $\hat{R}_{2}=\phi_{i}$ on $B_{i}$. There are two ways to do this.

First, $R_{2}$ is a quasiconformal conjugate of a rational map. Next, by an important result of Rickman, 15 , each rational map of $\overline{\mathbb{C}}$ has a quasiregular extension to a quasiregular map of $\overline{\mathbb{R}}^{3}$. A result of Carleson says each quasiconformal map of $\overline{\mathbb{C}}$ extends to a quasiconformal map of $\overline{\mathbb{R}}^{3}$, extending our conjugacy provides a means of extending $R_{2}$. Then we have to go about modifying this extension, using the annulus theorem again (this time in space and keeping $\overline{\mathbb{C}}$ invariant), so as to achieve 
the assertion that $\hat{R}_{2}$ is Möbius on the balls $B_{i}$ and still keep Condition (3) with $D_{i}$ replaced with $B_{i}$. The details are a little messy, but the construction is clear.

Alternatively, a direct construction of such an extension is possible using a slight modification (and repeated application) of the explicit construction given in [7. There the construction is the extension of a degree 2 rational map and the branch set is a circle, but it can be easily modified to give an extension of arbitrary degree rational map with two critical points so the branch set of the extension is a circle. One obtains more general rational maps by combining these simpler mappings using the annulus theorem. In our situation we need to group together the balls with the same image. Indeed, this construction was given in a previous version of this paper; however, the details are quite long and there are technical complications.

Step 4 . The extension $\hat{R}_{2}$ is uniformly quasiregular in $\overline{\mathbb{R}}^{3}$ since Condition (3) is easily retained (for the balls $B_{i}$ replacing the disks $D_{i}$ ) and it implies, as before, that a forward orbit picks up at most a finite amount of distortion. Now the map $f_{2}$ has an extension to a mapping $F: \mathbb{S}^{3} \rightarrow \mathbb{S}^{3}, F \mid \overline{\mathbb{C}}=f_{2}$. Thus the map $\hat{R}=F^{-1} \circ \hat{R}_{2} \circ F$ is our extension. Since $\hat{R}_{2}$ is $u q r$, so is $\hat{R}$.

Corollary 3.1. Let $R$ be a rational mapping, all of whose critical points lie in the immediate basin of an attracting fixed point. Then $R$ admits an extension to $\mathbb{H}^{3}$ as a uqr-mapping, and hence is rational with respect to a bounded measurable conformal structure.

Proof. The map $R$ admits an inverse disk system.

Remarks.

1. It is not too difficult to see how to modify the construction so as to allow certain parabolic fixed points in $D_{0}$ (see [5]).

2. We have not been careful about control of the distortion of the extension and what it might depend on. This is because of examples of Mayer 13 which imply that only qualitative results are possible.

3. It also follows from the work of Mayer [12] that certain rational maps of Lattes̀ type admit $u q r$-extensions to $\mathbb{S}^{3}$. This is rather surprising in view of our nonexistence results to follow.

\section{FAtou AND Julia SETS}

In the sequel, all notions of continuity and convergence will be with respect to the chordal metric of $\mathbb{S}^{3}$. Let $f: \mathbb{S}^{3} \rightarrow \mathbb{S}^{3}$ be a uqr-mapping. Then the Fatou set of $f$ is defined as

$$
F(f)=\left\{x \in \mathbb{S}^{3} \text { : there is an open } U \ni x \text { and }\left\{f^{n}\right\}_{n=1}^{\infty} \mid U \text { is normal }\right\} .
$$

The Julia set of $f$ is $J(f)=\mathbb{S}^{3} \backslash F(f)$. Clearly the Fatou set is open and the Julia set is closed. If $f$ is not injective, then necessarily $\left\{f^{n}\right\}_{n=1}^{\infty}$ is an infinite collection of mappings.

It is more or less immediate from the definition that the Fatou and Julia sets are completely invariant. That is,

$$
f(F(f))=f^{-1}(F(f))=F(f) \quad \text { and } \quad f(J(f))=f^{-1}(J(f))=J(f) .
$$

A simple degree argument analogous to the classical case of iteration of a rational function (see [1] and [2]) shows that $\left\{f^{n}\right\}_{n=1}^{\infty}$ cannot be normal on the entire Riemann sphere if the branch set $B_{f} \neq \emptyset$. Thus the Julia set is never empty when 
the degree of $f$ is at least 2. Indeed the Julia set is closed and perfect, therefore uncountable. We will use this fact without comment in the sequel. We recall the following version of Rickman's theorem concerning normal families of quasiregular mappings.

Theorem 4.1. Let $\Omega \subset \mathbb{S}^{3}$ be a domain whose boundary has infinite cardinality. Suppose that $f_{j}: \Omega \rightarrow \Omega$ is a sequence of $K$-quasiregular mappings. Then $\left\{f_{j}\right\}$ is normal and if $f: \Omega \rightarrow \mathbb{S}^{3}$ is the local uniform limit of a subsequence of the $\left\{f_{j}\right\}$, then either $f$ is a quasiregular mapping $f: \Omega \rightarrow \Omega$ or $f$ is a constant mapping. This constant lies in $\bar{\Omega}$.

\section{Nonexistence of extensions}

In this section we shall prove various nonexistence results for an extension of a rational map $R$ which show in a certain sense that our existence theorem is nearly optimal.

Lemma 5.1. Let $R \in \mathcal{R}_{d}$ and let $\hat{R}$ be a uqr-extension of $R$ to $\mathbb{S}^{3}$. Then

$$
J(\hat{R})=J(R) .
$$

Proof. It is immediate that $J(R) \subset J(\hat{R})$. To see the converse, let $\Omega$ be any open set in the complement of $J(R)$. Since $\overline{\mathbb{C}}$ is completely invariant, we see that the sets $\hat{R}^{n}(\Omega)$ omit $J(R)$. This set is infinite; therefore, Rickman's normality criterion implies that $\left\{\hat{R}^{n}\right\}_{n=1}^{\infty}$ is normal on $\Omega$.

Next, we would like to acknowledge L. Geyer for help with the proof of the following theorem, which considerably simplifies and extends an earlier version.

Theorem 5.1. Let $R$ be a rational map whose Julia set separates the plane. Then $R$ admits a uqr-extension only if the iterates of $R$ have a single constant limit function.

Proof. Let $\hat{R}$ be a $u q r$-extension of $R$. Set $\Omega=\mathbb{S}^{3} \backslash J(R)$ as the Fatou set of $\hat{R}$. Let us first suppose that there is a nonconstant limit function. Then of course Theorem 4.1 implies that $\left\{\hat{R}^{n}\right\}$ also has a nonconstant limit function, say $\hat{R}^{n_{j}} \rightarrow H$, $H: \Omega \rightarrow \mathbb{S}^{3}$ quasiregular. In particular, we shall use that $H$ is open. Suppose that $U \subset \mathbb{C}$ is a component of the Fatou set of $R$ which is not periodic. As there are nonconstant limit functions, such a component exists (see [1] and 2]). Then there is an open set $V \subset \mathbb{C}$ such that $H(V)=U$. To see this, note that $\Omega$ is a connected domain. If $H(\Omega)$ omits $U$, then there is $y \in \partial H(\Omega) \cap \Omega$. The preimage $x$ of $y$ lies in $\Omega$ as the Fatou set is completely invariant, but then $H$ cannot be open, as the image of a neighbourhood of $x$ is not a neighbourhood of $y$. But now $H(V)=U$ is also impossible, for if $z \in V$,

$$
F(R) \supset U \ni H(z)=\lim _{j \rightarrow \infty} \hat{R}^{n_{j}}=\lim _{j \rightarrow \infty} R^{n_{j}} \notin U \subset F(R)
$$

as the forward orbits accumulate only in the periodic components by Sullivan's no wandering domains theorem.

We deduce that $R$ has no nonconstant limit functions. The map $R$ has finitely many periodic components, and it requires only a little argument to see that we may assume that $R$ fixes each of them. From the classification of invariant components, each nonconstant limit function is an attracting or indifferent fixed point. In any 
case, we choose points $z_{0}$ and $z_{1}$ in different invariant components. Note that while $z_{0}$ and $z_{1}$ are in different components of $F(R)$, they are in the same component of $F(\hat{R})$ and therefore must have the same constant limit under iteration. We deduce every limit function is the same constant.

We next consider the case of super-attracting fixed points.

Theorem 5.2. Let $R \in \mathcal{R}_{d}$ and suppose that $R$ has a super-attracting fixed point. Then $R$ has no uqr-extension.

Proof. We may conjugate $R$ by a quasiconformal mapping $f$ of $\overline{\mathbb{C}}$ so that 0 is the super-attracting fixed point, $D=\{z:|z|<1\}$ lies in the immediate basin of 0 and that on the disk $D$ we have $S(z)=f \circ R \circ f^{-1}(z)=z^{k}$, for some $k \geq 2$. It is only necessary to show that $S$ does not have a uqr-extension.

Suppose that $S$ has such an extension $\hat{S}$. Let $\Omega=F(\hat{S})$, the Fatou set of $\hat{S}$, and recall that $\Omega$ is a normal domain in the sense of [14, page 18. Since the Julia set is closed and perfect, there is a neighbourhood $U$ of the unit disk in $\overline{\mathbb{R}}^{3}$ which lies in the Fatou set of $\hat{S}$. Let $E$ denote the component of the branch set of $\hat{S}$ containing 0 . Then $E$ is a nondegenerate continuum. Next, because of normality in $U$, the inverse images of $E$ under the iterates of $\hat{S}$ do not have an accumulation point in $D$, other than 0 . Therefore there is a neighbourhood $V$ of the circle $\{|z|=1 / 2\}$ which does not meet any inverse image of $E$. Let $\Gamma_{n}$ be the curve family consisting of curves in $\Omega$ joining the circle $\{|z|=1 / 2\}$ to the component $E_{n} \subset \hat{S}^{-n}(E)$ containing 0 . Since these images are nondegenerate continua containing 0 and exiting $U$ (indeed $E_{n}$ must accumulate on $\left.J(\hat{R})\right)$ we see that there is an absolute constant $A$, such that, for all $n$,

$$
M\left(\Gamma_{n}\right)<A,
$$

where $M\left(\Gamma_{n}\right)$ denotes the modulus of the curve family $\Gamma_{n}$. Now we look at the "images" of $\Gamma_{n}$ under $\hat{S}^{n}$. That is, the curve families $\Gamma_{n}^{\prime}$ joining the circle $\left\{|z|=2^{-k^{n}}\right\}$ to $E$ in $\Omega$. A simple consequence of the extremality of the Teichmüller ring shows that there is an absolute constant $a>0$ such that for all $n$,

$$
M\left(\Gamma_{n}^{\prime}\right) \geq a .
$$

Since $\hat{S}$ is $u q r$, the inner dilatation of the mapping $\hat{S}^{n}$ is bounded above by a constant, say $K$. The hypotheses of Väisälä's inequality ([14], Corollary 9.2) are now satisfied and we see that

$$
M\left(\Gamma_{n}^{\prime}\right) \leq \frac{K}{k^{n}} M\left(\Gamma_{n}\right) .
$$

And so, in particular,

$$
a \leq \frac{K}{k^{n}} A,
$$

which is not possible for $n$ sufficiently large. This contradiction establishes the result. 
Corollary 5.1. Let $R \in \mathcal{R}_{d}$ be a rational mapping. Suppose one of the following occurs.

(1) For some $N, R^{N}$ has at least two fixed points which are either attracting or rationally indifferent.

(2) $R$ has a Siegel disk or Herman ring or a cycle of such.

(3) $R$ has a super-attracting cycle.

Then there is no uqr-extension of $R$ to $\mathbb{S}^{3}$. In particular, polynomials do not admit uqr-extensions.

The reader will no doubt be aware that we have not discussed extension in the case that the Julia set is a nondegenerate nonseparating continuum in the plane. There it appears that virtually nothing is known. It is clear that the set of rational maps which admit a $K$-uqr-extension is closed and this presents the possibility that some such maps might be found to extend if one could get control of the distortion of the extension constructed in the first section. However, this seems beyond the scope of current methods. There is still a further case we have not considered. That is when the Julia set is a circle with a single rationally indifferent fixed point. There is no known example of a $u q r$ map of $\mathbb{S}^{3}$ whose Julia set is a circle. This leads us to ask

Question. Does the rational map $z \mapsto z+1 / z$ admit a $u q r$-extension?

\section{REFERENCES}

[1] A. Beardon, Iteration of Rational Functions, Springer-Verlag, 1991. MR1128089 (92j:30026)

[2] L. Carleson and T. Gamelin, Complex Dynamics, Springer-Verlag, $1993 . \quad$ MR1230383 (94h:30033)

[3] A. Hinkannen, Semigroups of planar quasiregular mappings, Ann. Acad. Sci. Fenn. Ser. A I Math.

[4] A. Hinkannen and G. J. Martin, Attractors in quasiregular semigroups, Proc. XVI Nevanlinna colloquium, Eds. I. Laine and O. Martio, de Gruyter, Berlin-New York, 1996, 135-141. MR $1427078(97 \mathrm{~m}: 30028)$

[5] A. Hinkannen, G. J. Martin and V. Mayer, The dynamics of UQR-mappings, Math. Scand. (to appear).

[6] T. Iwaniec and G. J. Martin, Quasiregular Semigroups, Ann. Acad. Sci. Fenn. Math. 21 (1996), no. 2, 241-254. MR.1404085 (97i:30032)

[7] T. Iwaniec and G. J. Martin, Geometric Function Theory and Nonlinear Analysis, Oxford University Press, 2001. MR 1859913 (2003c:30001)

[8] G.J. Martin, Branch sets of uniformly quasiregular maps, Conform. Geom. Dyn. 1 (1997), 24-27. MF 1454921 (98d:30032)

[9] G.J. Martin and V. Mayer, Rigidity in holomorphic and quasiregular dynamics, Trans. Amer. Math. Soc. 355 (2003), no. 11, 4349-4363. MR1990755 (2004i:37095)

[10] O. Martio and U. Srebro, Automorphic quasimeromorphic mapping in $\mathbb{R}^{n}$, Acta Math. 135 (1975) 221-247. MR.0435388 (55:8348)

[11] R. Mañè, P. Sad and D. Sullivan, On the dynamics of rational maps, Ann. Sci. École Norm. Sup. 16 (1983) 193-217. MR0732343 (85j:58089)

[12] V. Mayer, Uniformly quasiregular mappings of Lattès type, Conform. Geom. Dyn. 1 (1997), 104-111. MR 1482944 (98j:30017)

[13] V. Mayer, Behaviour of quasiregular semigroups near attracting fixed points, Ann. Acad. Sci. Fenn. Math. 25 (2000), 31-39. MR1737425|(2000k:30029) 
[14] S. Rickman, Quasiregular Mappings, Springer-Verlag 1993. MR1238941 (95g:30026)

[15] S. Rickman, The analogue for Picard's theorem for quasiregular mappings in dimension 3, Acta Math. 154 (1985), 195-242. MR0781587 (86h:30039)

Department of Mathematics, University of Auckland and Massey University, AuckLAND, NeW ZEALAND

E-mail address: martin@math.auckland.ac.nz 